\title{
Analytical Model for Message Propagation in Delay Tolerant Vehicular Ad Hoc Networks*
}

\author{
Ashish Agarwal, David Starobinski and T.D.C. Little \\ Department of Electrical and Computer Engineering \\ Boston University, Boston, Massachusetts \\ $\{$ ashisha,staro,tdcl\}@bu.edu
}

May 14, 2008

MCL Technical Report No. 05-14-2008

\begin{abstract}
In this paper we present an analytical model for delay tolerant message propagation in a dynamic vehicular network. The analysis provides upper and lower bounds for message propagation as function of traffic density, vehicle speed and radio range. The model is an extension of previous work which considered a particular network setting. The results from the analytical model are compared with simulation results for various vehicular traffic densities. The work demonstrates that increased mobility of vehicles actually aids in messaging contrary to the expectation that it would be a hindrance due to frequent topology changes. An increase in vehicle speed from $0 \mathrm{~m} / \mathrm{s}$ to $20 \mathrm{~m} / \mathrm{s}$ results in a corresponding increase in message propagation rate of $200 \mathrm{~m} / \mathrm{s}$ for vehicular density of 25 vehicles $/ \mathrm{km}$.
\end{abstract}

\footnotetext{
*In Proc. IEEE Vehicular Technology Conference 2008, Singapore, May 2008. This work is supported by the NSF under grant No. CNS-0435353. Any opinions, findings, and conclusions or recommendations expressed in this material are those of the author(s) and do not necessarily reflect the views of the National Science Foundation.
} 


\section{Introduction}

Inter-vehicle communication is targeted to enable applications such as accident avoidance messaging, congestion sensing, traffic metering, and general purpose Internet access. Vehicular networking is characterized by vast fabric of roadways and large number of vehicles that ply on these roads. Providing complete connectivity to the network is challenging in terms of infrastructure requirements. The network topology changes at a fast pace as vehicles move on the roads. Furthermore, different applications are likely to have different requirements. Safety applications are expected to be low data-rate applications, confined to a small neighborhood of each vehicle but with strict latency constraints. Traffic related services are expected to have more relaxed latency constraints but are likely to require communication within neighborhoods spanning several kilometers. Internet access would require connectivity to the backbone Internet with the help of deployed infrastructure. The majority of applications described are localized in nature, meaning that data are often originated and consumed within a geographical area. The observation that data bear spatialtemporal correlation suggests that a decentralized solution is a more efficient approach towards deploying applications. However, due to the large size of the road network and varying vehicular traffic densities, the network is likely to be partitioned frequently and intermittently as the topology changes.

In this paper, we consider a model to study the dissemination of safety messaging in a VANET (Vehicular Ad Hoc Network) absent any fixed road-side infrastructure. We base the model on a simple broadcast based dissemination scheme proposed elsewhere [1]. The scheme relies on attributed (labeled) data and map information such as GPS coordinates to disseminate data away from the point of incidence. The model is an extension to previous work which considered a particular network setting [2]. In this work, we generalize the model for a delay tolerant network as a function of vehicular traffic density on either side of the roadway, radio range and vehicle speed. We compare the upper and lower bounds derived with simulation results for a set of parameters.

The rest of the paper is organized as follows - Section II describes the related work in this area. In Section III, we describe the routing scheme and VANET scenario. The analytical model is presented in Section IV followed by the simulation results in Section V. Finally, we summarize our results in Section VI.

\section{Related Work}

The IEEE WAVE (Wireless Access for Vehicular Environments) [3] is a group dedicated to development of the 802.11 p draft to enable vehicle-to-vehicle (V2V) and vehicle-to-infrastructure (V2I) safety communication. Vehicle-to-vehicle communications has been explored in projects such as FleetNet and CarTALK [4]. However, the approach in these projects assumes complete connectivity of the network. We instead focus on cases in which data can tolerate some delay as would be expected in an intermittently connected network. We use a model characterized by constant fragmentation due to transiting blocks of vehicles.

Delay tolerant networking (DTN) and custody transfer [5] are concepts that we apply here in conjunction with the use of labeled data. DTN is essentially a store-and-forward scheme that messages are stored in the memory of a node and forwarded whenever connectivity to the next hop is available in a partitioned network. As messaging is connectionless, accounting for acknowledg- 
ment of delivery is difficult. The custody transfer mechanism permits exchange of responsibility for forwarding messages from one node to the next in forward progress.

The UMass DieselNET project explores the deployment of communication infrastructure over the campus transportation network and record measurements on opportunistic networking [6]. Wu et al. have proposed an analytical model to represent a highway-vehicle scenario [7]. In their approach, they investigate speed differential between vehicles traveling in the same direction to bridge partitioned network of vehicles. In our model, we demonstrate that the transient connectivity offered by opposing traffic can provide a substantial improvement in message propagation rate.

\section{VANET Scenario and Routing Scheme}

We concentrate on the problem of information propagation on a roadway without deployed infrastructure. The roadway is modeled as rectilinear, as illustrated in Figure 1, and that packet radio is tolerant to local variations in directionality and curvature of the roadway. Vehicles are equipped with sensing, communication, and computing capabilities and form nodes of an infrastructure-less ad hoc network.

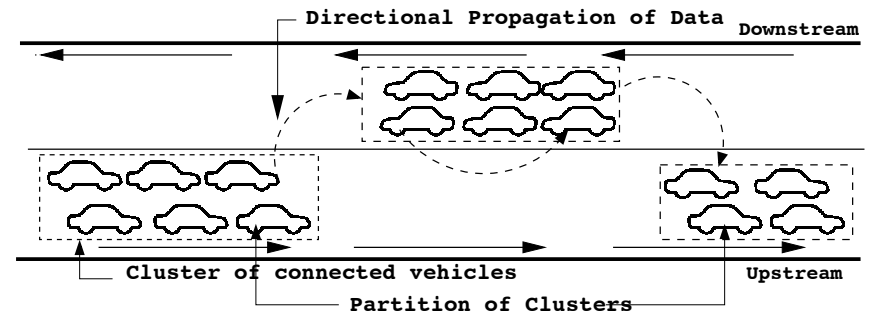

Figure 1: Illustration of fragmented highway scenario.

A time-series of snapshots of vehicular traffic data demonstrates that vehicles are grouped in small disconnected clusters $[4,8]$. In networking terminology, the connectivity graph formed by vehicles can be described as a partition yielding multiple disconnected subnets. Formation of end-to-end paths is difficult, if not impossible, especially in low vehicular density scenarios. If nodes traveling in opposing direction are used in path formation, the resulting paths are shortlived, leading to considerable overheads in dynamic path formation and route maintenance. Thus, MANET routing protocols which rely on such strategies are a poor solution.

We consider a routing scheme of the form proposed in reference [1]. This scheme is based on message labeling using descriptive tags supporting the message propagation goal. For example, with the availability of location and map information from GPS devices in vehicles, messages can be attributed with source and destination locations or regions. These tags, or attributes, work in conjunction with a message time-to-live (TTL), a function of time and area, that provides control over the extent of dissemination away from a traffic incident. To bridge traffic clusters (fragments) a custody transfer scheme is adopted from the delay tolerant networking [5].

Messages and vehicles travel either upstream or downstream relative to their counterparts. Upstream messages are forwarded by vehicles traveling upstream by exploiting commonality of direction. When a partition in upstream traffic is encountered, messages can be forwarded via downstream traffic to exploit possible connectivity there. This downstream traffic is, in some cases, 
sufficient to bridge the partition. With the help of attributed data, the protocol is able to maintain the directional propagation of data even with transient connectivity. Thus, this scheme exploits time-varying connectivity in an opportunistic way to bridge partitions (Figure 1). Our main focus in this paper is to characterize this behavior.

\section{Analytical Model}

Consider a highway scenario as described in Section III, a simplified model of a vehicular highway as nodes located randomly along a line. Nodes traveling in one direction are separated by distances $X=\left\{X_{i}, i=1,2, \ldots\right\}$, where $X$ denotes i.i.d. random variables that are exponentially distributed. Given a fixed transmission range $R>0$, two nodes (vehicles) are connected if $X_{i} \leq R$. We assume that vehicle size (length) to be negligible in terms of its impact on connectivity.

Vehicles on a roadway are traveling in either upstream or downstream direction, as illustrated in Figure 1. The inter-vehicular distance is distributed as an exponential random variable with parameter $\lambda_{u}$ and $\lambda_{d}$ for upstream and downstream traffic respectively. An exponential distribution is chosen to leverage the memory-less property to ease the analysis. The distribution at any point of the highway is independent of the preceding or succeeding traffic on the roadway. Like-wise, the connectivity is modeled as the probability that the inter-node distance is less than or equal to the transmission range $R$, numerically $P\left(X_{i}<R\right)$.

For the purpose of analysis, the length of the roadway on either side is divided into cells of size $l$ (Figure 2). We define two bounds for the cell size; $R$, an upper bound, and $R / 2$, a lower bound. The bounds are defined such that, for the lower bound, two nodes located in adjacent cells are surely connected as the maximum distance between the two nodes is $R$. However, for the upper bound cell size $R$, the distance between two nodes is such that $\left(0 \leq X_{i} \leq 2 R\right)$. Thus, with cell size $R$, the nodes are not necessarily connected. However, there are possible arrangements where the nodes could be connected as illustrated in Figure 2 . At the lower bound $R / 2$, the nodes are always connected, however, there are cases in which there are more nodes than are required to achieve connectivity. Thus, the upper bound is a necessary but not sufficient condition. Whereas, the lower bound is a sufficient but not always necessary condition.

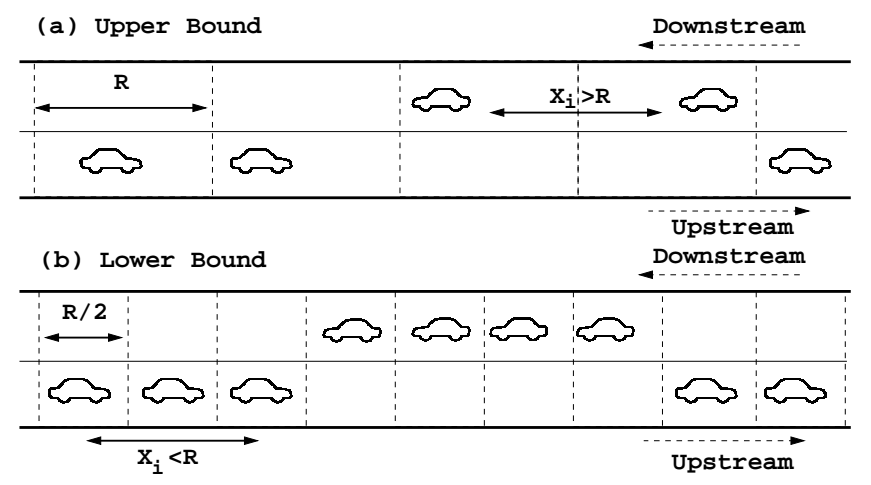

Figure 2: Illustration of upper and lower bounds.

The vehicles, in both directions, are assumed to move at a constant speed of $v \mathrm{~m} / \mathrm{s}$ such that the inter-node distance along a direction remains unchanged. We define a data propagation speed 
$v_{\text {radio }} \mathrm{m} / \mathrm{s}$, as determined by the characteristics of the packet radio physical layer. It is approximated by the maximum distance propagated in one second, including intermediate hops. For simplicity, we assume that $v<<v_{\text {radio }}$, that is, data travel much faster than vehicles.

One metric to estimate the performance of the network is the average data propagation rate in the given topology. With the assumption of delay tolerance in the network, data are buffered at nodes until connectivity is available. Thus, the average rate of data propagation is a function of the connectivity. We call the alternating periods of multi-hop connectivity and disconnection as Phase 1 and Phase 2, respectively. In Phase 1, nodes are connected, data propagate multi-hop propagation rate $v_{\text {radio }} \mathrm{m} / \mathrm{s}$. While in Phase 2 , nodes are disconnected, the data propagate at vehicle speed $(v \mathrm{~m} / \mathrm{s})$ in buffered mode until connectivity. We define $v_{e f f}$ as the effective propagation rate which is a function of the time and distance in the two phases.

Theorem 4.1 The effective propagation rate $v_{\text {eff }}$ lies between the bounds: $v_{\text {low }} \leq v_{\text {eff }} \leq v_{u p}$; where $v_{u p}$ is an upper bound on the propagation rate, given by:

$$
v_{u p}=\frac{E\left[D_{1}\right]_{u p}+E\left[D_{2}\right]_{u p}}{\frac{E\left[D_{1}\right]_{u p}}{v_{\text {radio }}}+\frac{E\left[D_{2}\right]_{u p}}{v}+\frac{E[X \mid \bar{C}]}{v_{\text {radio }}}}
$$

and $v_{\text {low }}$ is a lower bound given by:

$$
v_{\text {low }}=\frac{E\left[D_{1}\right]_{\text {low }}+E\left[D_{2}\right]_{\text {low }}}{\frac{E\left[D_{1}\right]_{\text {low }}}{v_{\text {radio }}}+\frac{E\left[D_{2}\right]_{\text {low }}}{v}+\frac{E[X \mid \bar{C}]}{v_{\text {radio }}}}
$$

where $E\left[D_{1}\right]_{\text {up }}, E\left[D_{2}\right]_{\text {up }}, E\left[D_{1}\right]_{\text {low }}$ and $E\left[D_{2}\right]_{\text {low }}$ are the upper and lower bound values of expected distances covered in Phases 1 and 2 respectively. Expressions for these quantities are given by Equations (14),(15),(16),(17).

Proof: For the sake of brevity, we will derive the upper bound (cell size R) for $v_{\text {eff }}$ and suitably substitute for deriving the expression for the lower bound (cell size $\mathrm{R} / 2$ ). We derive the propagation rates in Phase 1 and Phase 2 for the upper bound and extend the results for the lower bound. Due to space constraint, we describe the proof concisely in this text and refer to [9] for details.

In Phase 1, the nodes are connected and data are able to propagate multi-hop. As illustrated in Figure 3, when the nodes upstream are disconnected the messages are forwarded by nodes traveling in the downstream direction to the next hop upstream. If two adjacent nodes along upstream are

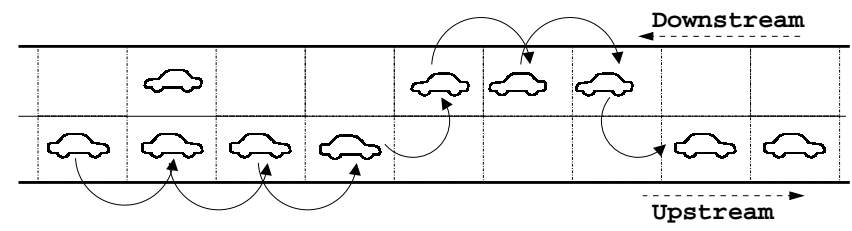

Figure 3: Illustration of connectivity in Phase 1 of data propagation.

not connected by a direct link (i.e. $X_{i}>R$ ), then connectivity must be achieved using nodes along downstream direction. Consider the distance between upstream nodes to be divided into cells as illustrated in Figure 3. They are connected if each cell along the gap $X_{i}$ is occupied by at least one 
node, which occurs with probability $p=\left(1-e^{-\lambda_{d} R}\right)^{\lfloor x / R\rfloor}$. The probability of connectivity $(C)$ for given value of inter-node distance $(x)$ can be expressed as:

$$
P(C \mid X=x)= \begin{cases}1 & \text { if } x \leq R, \\ \left(1-e^{-\lambda_{d} R}\right)^{\lfloor x / R\rfloor} & \text { if } x>R\end{cases}
$$

The probability of connectivity for nodes along upstream traffic $P(C)$, independent of inter-node distance:

$$
P(C)=\int_{0}^{\infty} P(C \mid X=x) f_{X}(x) d x=\frac{\left(1-e^{-\lambda_{u} R}\right)}{1-e^{-\lambda_{u} R}\left(1-e^{-\lambda_{d} R}\right)}
$$

Given connectivity, the density function for data propagation distance is expressed as:

$$
\begin{aligned}
& f_{X \mid C}(x)=\quad \frac{1}{P(C)} f_{X}(x) P(C \mid X=x) \\
& = \begin{cases}\frac{\left(\lambda_{u} e^{-\lambda_{u} x}\right)}{P(C)} & \text { if } x \leq R \\
\frac{\left(\lambda_{u} e^{-\lambda_{u} x}\left(1-e^{-\lambda_{d} R}\right)^{\lfloor x / R\rfloor}\right)}{P(C)} & \text { if } x>R\end{cases}
\end{aligned}
$$

Thus, the expected distance of data propagation in Phase 1 is evaluated as:

$$
\begin{gathered}
E[X \mid C]=\int_{0}^{\infty} x f_{X \mid C}(x) d x=\int_{0}^{\infty} \frac{x f_{X}(x) P(C \mid X=x) d x}{P(C)} \\
E[X \mid C]=\frac{1}{P(C)} \frac{1}{\lambda_{u}}\left[\frac{1-\left(1+\lambda_{u} R\right) e^{-\lambda_{u} R}}{1-e^{-\lambda_{u} R}\left(1-e^{-\lambda_{d} R}\right)}+\ldots\right. \\
\left.+\frac{\lambda_{u} R e^{-\lambda_{u} R}\left(1-e^{-\lambda_{d} R}\right)\left(1-e^{-\lambda_{u} R}\right)}{\left(1-e^{-\lambda_{u} R}\left(1-e^{-\lambda_{d} R}\right)\right)^{2}}\right]
\end{gathered}
$$

The expression for expected distance in Phase 1, independent of connectivity $(\mathrm{C})$ is evaluated as:

$$
E\left[D_{1}\right]_{u p}=\frac{E[X \mid C] P(C)}{1-P(C)}
$$

Substituting for $E[X \mid C]$ and $P(C)$ from Equations (6) and (4) respectively, we obtain the final expression for the upper bound for expected distance in phase 1 in (14).

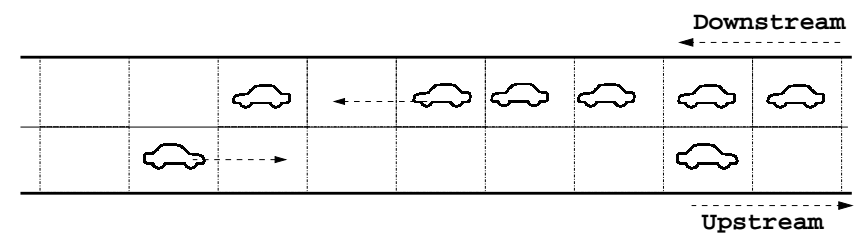

Figure 4: Illustration of connectivity in Phase 2 of data propagation.

In Phase 2, there is an absence of multi-hop connectivity in upstream and downstream traffic (Figure 4). We denote the number of cells in the gap $(x)$ to be $k=x / R$. The data are cached 

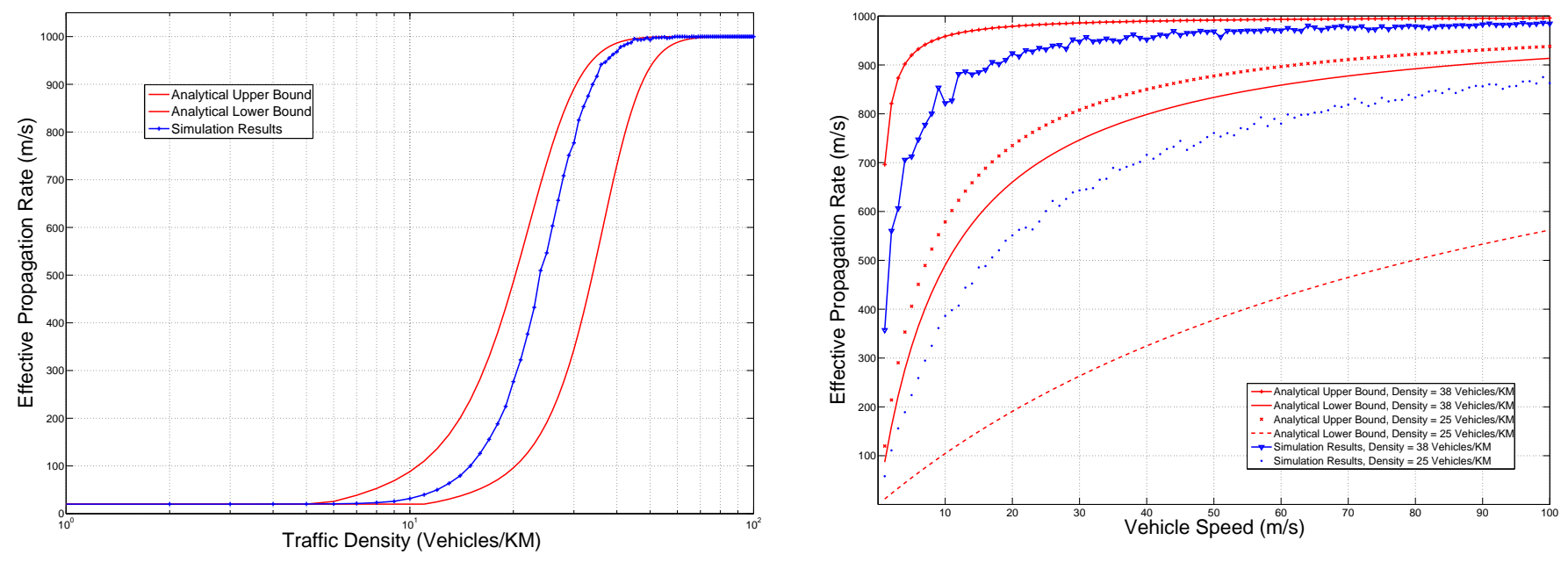

Figure 5: Simulation results : (a) Message Propagation rate as density in the network increases. (b) Message propagation rate as vehicle speed increases at fixed density.

until connectivity is achieved, i.e., all $k$ cells in the gap have at least one node. Until connectivity, the node traverses distance at a speed of $v \mathrm{~m} / \mathrm{s}$. The number of cells traversed until connectivity is analogous to the number of trials until a sequence is seen, described as pattern matching in classical probability theory [10]. The pattern matching problem describes the task to compute the expected number of trials until $k$ consecutive successes, or $k$ consecutive cells along the downstream traffic to be occupied by nodes. From known results on pattern matching [10], the expected number of trials, $N$, traversed until $k$ consecutive successes is: $\sum_{i=1}^{k} 1 / p^{i}$ or equivalently, $E[N]=\frac{\left(1-p^{k}\right)}{(1-p) p^{k}}$ where $p$ is the probability of success for a single event.

For the upper bound cell size $(R)$, we compute the distance traversed until the requisite connectivity. The data are propagated a distance equivalent to $E[N]-k$ cells at speed of the node $(v \mathrm{~m} / \mathrm{s})$, and a distance $k$ cells at multi-hop speed $\left(v_{\text {radio }} \mathrm{m} / \mathrm{s}\right.$ ) once connectivity is achieved. Since traffic in both directions is moving at the same speed, the distance is adjusted by a factor of $1 / 2$. Thus, the expected distance, for a given separation $\left(X_{i}=x\right)$, traversed by node the before connectivity is available is given by:

$$
\frac{(E[N]-k) R}{2}=\left(\frac{\left(1-p^{k}\right)}{(1-p) p^{k}}-k\right)\left(\frac{R}{2}\right)
$$

The probability of success is equivalent to probability of connectivity along downstream traffic, i.e., $p=\left(1-e^{-\lambda_{d} R}\right)$. We consider the upper bound value $k=\lfloor x / R\rfloor$. This is an upper bound as the floor function gives the greatest integer value of cells less than $x / R$. Thus, the expected distance of data propagation, for given node separation, in Phase 2 is:

$$
E\left[D_{2} \mid X=x\right]=\left[\frac{1-p^{\lfloor x / R\rfloor}}{(1-p) p^{\lfloor x / R\rfloor}}-\left\lfloor\frac{x}{R}\right\rfloor\right] \frac{R}{2}
$$

In Phase 2, there is absence of connectivity along either upstream or downstream nodes. Thus, the 


$$
\begin{aligned}
E\left[D_{1}\right]_{u p} & =\frac{1}{\lambda_{u}}\left[\frac{1-e^{-\lambda_{u} R}\left(1-e^{-\lambda_{d} R}\right)}{e^{-\lambda_{u} R} e^{-\lambda_{d} R}}\right]\left[\frac{1-\left(1+\lambda_{u} R\right) e^{-\lambda_{u} R}}{1-e^{-\lambda_{u} R}\left(1-e^{-\lambda_{d} R}\right)}+\frac{\lambda_{u} R e^{-\lambda_{u} R}\left(1-e^{-\lambda_{d} R}\right)\left(1-e^{-\lambda_{u} R}\right)}{\left(1-e^{-\lambda_{u} R}\left(1-e^{-\lambda_{d} R}\right)\right)^{2}}\right] \\
E\left[D_{2}\right]_{u p} & =\frac{R\left(1-e^{\lambda_{u} R}\right)}{2 P(\bar{C})}\left[\frac{1}{1-p}\left[\frac{e^{-\lambda_{u} R}}{p-e^{-\lambda_{u} R}}+\frac{p e^{-\lambda_{u} R}}{1-p e^{-\lambda_{u} R}}-\frac{2 e^{-\lambda_{u} R}}{1-e^{-\lambda_{u} R}}\right]-\frac{e^{-\lambda_{u} R}}{\left(1-e^{-\lambda_{u} R}\right)^{2}}+\frac{p e^{-\lambda_{u} R}}{\left(1-p e^{-\lambda_{u} R}\right)^{2}}\right] \\
E\left[D_{1}\right]_{\text {low }} & =\frac{1}{\lambda_{u}}\left[\frac{1-e^{-\lambda_{u} R}\left(1-e^{-\lambda_{d} R}\right)}{e^{-\lambda_{u} R_{e}} e^{-\lambda_{d} R / 2}}\right]\left[1-e^{-\lambda_{u} R}\left(1+\lambda_{u} R\right)+\frac{\lambda_{u} \frac{R}{2}\left(e^{-\lambda_{u} R / 2}\right)^{2}\left(1-e^{-\lambda_{d} R / 2}\right)^{2}\left(1-e^{-\lambda_{u} R / 2}\right)}{\left(1-e^{-\lambda_{u} R / 2}\left(1-e^{-\lambda_{d} R / 2}\right)\right)^{2}}\right] \\
E\left[D_{2}\right]_{\text {low }} & =\frac{R\left(1-e^{\lambda_{u} \frac{R}{2}}\right)}{4 P(\bar{C})}\left[\frac{1}{1-p}\left[\frac{e^{-\lambda_{u} \frac{R}{2}}}{p-e^{-\lambda_{u} \frac{R}{2}}}+\frac{p e^{-\lambda_{u} \frac{R}{2}}}{1-p e^{-\lambda_{u} \frac{R}{2}}}-\frac{2 e^{-\lambda_{u} \frac{R}{2}}}{1-e^{-\lambda_{u} \frac{R}{2}}}\right]-\frac{e^{-\lambda_{u} \frac{R}{2}}}{\left(1-e^{-\lambda_{u} \frac{R}{2}}\right)^{2}}+\frac{p e^{-\lambda_{u} \frac{R}{2}}}{\left(1-p e^{-\lambda_{u} \frac{R}{2}}\right)^{2}}\right]
\end{aligned}
$$

probability that nodes are disconnected $(\bar{C})$, given inter-node separation is:

$$
P(\bar{C} \mid X=x)= \begin{cases}0 & \text { if } x \leq R, \\ 1-\left(1-e^{-\lambda_{d} R}\right)^{\lfloor x / R\rfloor} & \text { if } x>R\end{cases}
$$

The probability nodes are disconnected $(\bar{C})$ independent of node distribution is evaluated as complimentary of the probability of connectivity (Eqn (4)):

$$
P(\bar{C})=1-P(C)=\frac{e^{-\lambda_{u} R} e^{-\lambda_{d} R}}{1-e^{-\lambda_{u} R}\left(1-e^{-\lambda_{d} R}\right)}
$$

The density function is evaluated as:

$$
\begin{aligned}
f_{X \mid \bar{C}}(x) & =\frac{f_{X}(x) P(\bar{C} \mid X=x)}{P(\bar{C})} \\
& = \begin{cases}0 & \text { if } x \leq R \\
\frac{\left(\lambda_{u} e^{-\lambda_{u} x}\left(1-\left(1-e^{-\lambda_{d} R}\right)^{\lfloor x / R\rfloor}\right)\right)}{P(\bar{C})} & \text { if } x>R\end{cases}
\end{aligned}
$$

Generalizing the result in Equation (9):

$$
\begin{aligned}
E\left[D_{2}\right]_{u p} & =\int_{0}^{\infty} E\left[D_{2} \mid X\right] f_{X \mid \bar{C}}(x) d x \\
& =\int_{R}^{\infty} \frac{E\left[D_{2} \mid X\right] f_{X}(x) P(\bar{C} \mid X=x)}{P(\bar{C})} d x
\end{aligned}
$$

The final expression is given in Equation (15). Similarly, for the lower bound cell size $(R / 2)$, we derive the corresponding expressions in Equations (16) and (17).

Finally, the expected size of gap in Phase 2 is:

$$
E[X \mid \bar{C}]=\int_{R}^{\infty} x f_{X \mid \bar{C}}(x) d x=\int_{R}^{\infty} \frac{x f_{x}(x) P(\bar{C} \mid X=x)}{P(\bar{C})}
$$




\section{Performance Results}

We compare the analytical model with simulations for a set of parameters; radio range $R=125 \mathrm{~m}$, vehicle speed $v=20 \mathrm{~m} / \mathrm{s}$, radio propagation speed $v_{\text {radio }}=1000 \mathrm{~m} / \mathrm{s}$. The traffic density is varied from 1 vehicle $/ \mathrm{km}$ to 100 vehicles/ $\mathrm{km}$ to cover the low, intermediate and high traffic scenarios. Figure 5(a) shows the simulation of message propagation rate at various densities lies well within the upper and lower bounds defined by analytical model. As described earlier, the message dissemination lies predominantly in two regimes, one - where the network is fully connected, the propagation rate is at a maximum $v_{\text {radio }} \mathrm{m} / \mathrm{s}$, second - where nodes in the network are partitioned and messages are disseminated as the vehicles moves at vehicle speed $(v \mathrm{~m} / \mathrm{s})$. The interesting observation in this graph is the intermediate density case where opportunistic contacts achieve improved message propagation.

The graph in Figure 5(b) shows the analytical and simulation results for the message propagation rate at constant density as the vehicle speed is increased from $0 \mathrm{~m} / \mathrm{s}$ to $100 \mathrm{~m} / \mathrm{s}$. The graph depicts an order of magnitude increase in message propagation rate as vehicle speed is increased, at constant density. This result is counter-intuitive - one would expect an increase in traffic speed to create an increase frequency of disconnection, yet it results in increased message propagation rate. The magnitude of increase, however, depends upon the density of vehicles in the network. An increase in traffic speed from $0 \mathrm{~m} / \mathrm{s}$ to $20 \mathrm{~m} / \mathrm{s}$ results in a corresponding increase in message propagation rate from $0 \mathrm{~m} / \mathrm{s}$ to at least $200 \mathrm{~m} / \mathrm{s}$, at a vehicular density of 25 vehicles $/ \mathrm{km}$. Correspondingly, the message propagation rate increases from $0 \mathrm{~m} / \mathrm{s}$ to $600 \mathrm{~m} / \mathrm{s}$ for vehicular density of 37 vehicles $/ \mathrm{km}$.

\section{Conclusion}

We have presented a unique scenario of message propagation in an intermittently connected network formed by vehicles transiting a highway. We describe a routing model for message dissemination in such a highway scenario. Particularly, we study the delay tolerant model with intermittent connectivity. The model and observations are relevant for application deployment as varying network conditions of high and low vehicular traffic density demand reconfigurable parameters. Simulation studies indicate that the analytical model captures the qualitative behavior of the routing scheme and the impact of vehicular density and vehicular speed on the effective propagation rate. The analysis reveals the complex inter-relationship of radio range, vehicular traffic density and vehicular speed in a delay tolerant network at the limit for convergence for the delay tolerant assumption. The results imply that the message propagation rate experiences a phase transition behavior as a function of the vehicular traffic density. In our future work, we expect to analyze the phase transition behavior in detail with respect to the critical density as the analysis will suggest the required infrastructure support for vehicular network applications. Thus, the model can be adapted to study the deployment of roadside infrastructure to support inter-vehicle communication.

Furthermore, the analysis and simulation demonstrate that opportunistic connections and networking are able to significantly improve message propagation rates, contrary to expectation. An important insight from this work is that vehicular mobility leading to opportunistic contacts can be leveraged for message propagation. These observations support the arguments for the application of techniques adopted from delay tolerant and mobile ad hoc networking in future vehicular networks. 


\section{References}

[1] T. D. C. Little and A. Agarwal, "An Information Propagation Scheme for Vehicular Networks," in Proc. IEEE Intelligent Transportation Systems Conference (ITSC), Vienna, Austria, September 2005, pp. 155-160.

[2] A. Agarwal, D. Starobinski, and T. D. C. Little, "Exploiting Downstream Mobility to Achieve Fast Upstream Propagation," in Proc. of Mobile Networking for Vehicular Environments (MOVE) at IEEE INFOCOM 2007, Anchorage, AK, May 2007.

[3] I. Berger, "Standards for Car Talk," The Institute, March 2007.

[4] H. Füßler, M. Mauve, H. Hartenstein, D. Vollmer, and M. Käsemann, "Location Based Routing for Vehicular Ad Hoc Networks," in Proc. MOBICOM '02, Atlanta, Georgia, USA, September 2002.

[5] K. Fall, "A Delay-Tolerant Network Architecture for Challenged Internets," in Proc. of SIGCOMM '03. New York, NY, USA: ACM Press, 2003, pp. 27-34.

[6] J. Burgess, B. Gallagher, D. Jensen, and B. N. Levine, "MaxProp: Routing for Vehicle-Based Disruption-Tolerant Networks," in Proc. IEEE Conference on Computer Communications (INFOCOM), Barcelona, Spain, April 2006, pp. 1-11.

[7] H. Wu, R. Fujimoto, R. Guensler, and M. Hunter, "Analytical Models for Information Propagation in Vehicle-to-Vehicle Networks," in Proc. ACM VANET, Philadelphia, USA, October 2004.

[8] V. Naumov, R. Baumann, and T. Gross, "An Evaluation of Inter-Vehicle Ad Hoc Networks Based on Realistic Vehicular Traces," in MobiHoc '06: Proc. 7th ACM Intl. Symp. on Mobile Ad Hoc Networking and Computing, New York, NY, USA, 2006.

[9] A. Agarwal, “Analytical Model for Data Propagation in Vehicular Networks," MCL TR-0201-2008, Tech. Rep., Feb. 2008.

[10] S. M. Ross, Introduction to Probability Models. Academic Press.

[11] T. Nadeem, P. Shankar, and L. Iftode, "A Comparative Study of Data Dissemination Models for VANETs," in Third Annual International Conference on Mobile and Ubiquitous Systems: Networking \& Services, 2006, pp. 1-10.

[12] W. Zhao and M. H. Ammar, "Message Ferrying: Proactive Routing in Highly-Partitioned Wireless Ad Hoc Networks," in FTDCS '03: Proceedings of the The Ninth IEEE Workshop on Future Trends of Distributed Computing Systems. Washington, DC, USA: IEEE Computer Society, 2003, p. 308.

[13] H. Wu, R. Fujimoto, R. Guensler, and M. Hunter, "MDDV: A Mobility-Centric Data Dissemination Algorithm for Vehicular Networks," in Proc. 1st ACM Intl. Workshop on Vehicular Ad Hoc Networks (VANET), Philadelphia, PA, USA, 2004, pp. 47-56. 